\title{
PD-FUZZY CONTROL OF SINGLE LOWER LIMB EXOSKELETON FOR HEMIPLEGIA MOBILITY
}

\author{
Abdullah K Alshatti ${ }^{1}$ and M. O. Tokhi ${ }^{2}$ \\ ${ }^{1}$ Department of Automatic Control and System Engineering, \\ University of Sheffield, United Kingdom \\ ${ }^{2}$ School of Engineering, London South Bank University, United Kingdom
}

\begin{abstract}
This paper presents studies in the design and control of single leg exoskeleton for hemiplegia mobility in simulation environment. The exoskeleton is designed to support the affected side of the hemiplegia patient while the other leg functions normally. Hip, knee and ankle joints for both humanoid leg and exoskeleton of the affected side are controlled using PD-Fuzzy control to obtain the required natural torque to allow the exoskeleton to compensate for the deficiency in affected leg to achieve normal symmetric gait. The controller is implemented in MATLAB, and the system behaviour observed in Visual Nastran 4D (VN4D) during simulation. Simulation results show that the exoskeleton can support the humanoid with the required augmentation using the proposed design and control.
\end{abstract}

\section{KEYWORDS}

Assistive robotics, Fuzzy logic control, Hemiplegia mobility.

\section{INTRODUCTION}

The human locomotion process depends on collaboration between the neuromuscular and musculoskeletal systems for achieving symmetric gait cycle, and any condition of injury, illness or disease will affect the nature of human walking pattern [1]. Human walking disorder can be related to different types of physical conditions such as limb amputation, paralysis, disability and injury. Human walking disorder can result in reduced ability in performing daily life activities due to reduced functionality of muscles as in elderly people or due to medical or physical conditions in general. In order to provide better quality of life for people who experience gait disorder so as to perform their usual daily tasks, attempts have been made to develop various solutions to assist and support their locomotion. According to the world health organization, worldwide 250,000 to 500,000 suffer from spinal cord injury every year. Spinal cord injury (SCI) symptoms may include loss of full or partial body limb control and sensory functions, and one of the essential measures to improve SCI medical care and rehabilitation services is to provide assistive devices to enable SCI patients to perform their daily life activities, but only 5-15\% of people who live in low and middle-income countries can afford assistive devices [2]. Providing innovative solutions to people with mobility disorder becomes an interesting area for researchers. By utilising control algorithms, sensory systems and appropriate motors, assistive robotics can be one of the cutting-edge solutions to deliver the right amount of support needed to assist people with mobility disorder. 
This paper initially presents a brief background about exoskeleton applications. Then the approach for design and control of humanoid and exoskeleton model is described. Simulation results are then presented, discussed and conclusion are drawn. The work presented in this paper builds up on previous work of the authors [3].

\section{BACKGROUND}

Exoskeletons can be categorised into two main types, namely assistive exoskeletons and rehabilitation exoskeletons and the design mechanisms vary based on application, such as full or partial body and lower or upper extremity. The current exoskeleton designs include three types, namely performance exoskeletons, rehabilitation exoskeletons and assistive exoskeletons [4]. Performance exoskeletons are developed for supplementation of human physical ability to perform tasks such as lifting heavy weight, rehabilitation exoskeletons are developed to help people who suffer from neurological injury to retrieve their mobility and assistive exoskeletons are developed to benefit people with mobility disorders to perform daily tasks. Research in exoskeleton systems has intensified in the late 20th century and researchers have reported various novel models of wearable robotics, and exoskeleton has become a research focus in the field of robotics [5].

Exoskeletons and active orthosis are currently considered as viable human physical assistance and support devices. In the last few years several efforts have been made to integrate robotics technology to human body in order to improve human physical abilities for better performance and one of these efforts is the exoskeleton, also referred to as active orthosis. Late 1960s, "Hardiman" considered one of the earliest full body exoskeleton developed by General Electric in cooperation with Cornell University to augment wearer strength. "Hardiman" was hydraulically power-driven machine with 30 DOF [6]. Berkeley lower extremity exoskeleton (BLEEX) is one of the first autonomous load carrying exoskeletons with seven DOF in each leg with actuated joint supporting hip, knee and ankle while three DOF are unactuated on ankle and hip [7]. Exoskeletons are also considered in the medical field especially for rehabilitation and gait disorder enhancement. Berkeley Bionic designed assistive exoskeleton (eLEGS) to support lower limbs of paralyzed patients to stand up and walk by actuating hip and knee joints and with the ankle actuated by passive spring, using finite state machine to differentiate between the four states in stride cycle to obtain the walking movement [8]. Cyberdyne launched the HAL (hybrid assistive leg) series of exoskeletons. HAL-5 has been designed to physically strengthen healthy people and assist people with gait disorder to perform their daily tasks, where the assistive strategy determined by using controller based on activity of the hip and knee (flexion/extension) actuators and recording walking pattern of the operator. EMG, GRF, potentiometer, gyroscope and accelerometer sensors are used to feed the controller with the essential data [9].

Exoskeleton robotic developments are still considered by researchers as challenge with many technological issues. Lightweight, energy consumption and exoskeleton design aspects are essential to the operator adaptability and functionality, but more sensors and actuation used in the exoskeleton device will lead to more operating complexity and high power consumption [10]. In addition, the cost of available commercial exoskeletons is too high. Therefore, one of the challenges and motivations in exoskeleton developments is to make them cheaper and affordable by considering the technology selection and exoskeleton design.

\section{SYSTEM MODEL}

The exoskeleton and humanoid model presented in this paper consists of different joint motors, links, exoskeleton frame and the controller. With such complexity of the system, modelling and design method using finite element analysis (FEA) has been selected to analysis how the 
exoskeleton reacts to various forces, disturbances as well as different controllers. The software facilities used to design the humanoid model and exoskeleton model comprise Solid Works and Visual Nastran 4D software. MATLAB software is used to develop the controller and simulation of the whole system model by integrating Visual Nastran 4D with MATLAB Simulink. The humanoid model with exoskeleton on the right leg was used to replicate real human movements by using torque as input and orientation as output representing hip and knee joints positions by using Visual Nastran 4D meter and control features.

\subsection{Humanoid}

The humanoid model shown in Figure 1 is designed to simulate human body characteristics such as width, height and weight, and replicate human physical appearance and movements according to the anthropometric data given in [11]. The humanoid model represents $75 \mathrm{Kg}$ in weight and 1.7 $\mathrm{m}$ in height. Humanoid parts and limbs were designed in Solid Works then exported and assembled in Visual Nastran software. The assembly of the humanoid design is based on different aspects such as degree of freedom, type of joint and axes of rotation. Each body segment is connected by choosing the appropriate joint constraints to activate and control the humanoid motion during the simulation. The humanoid joints are designed to perform motion in the sagittal plane while other axes are locked.

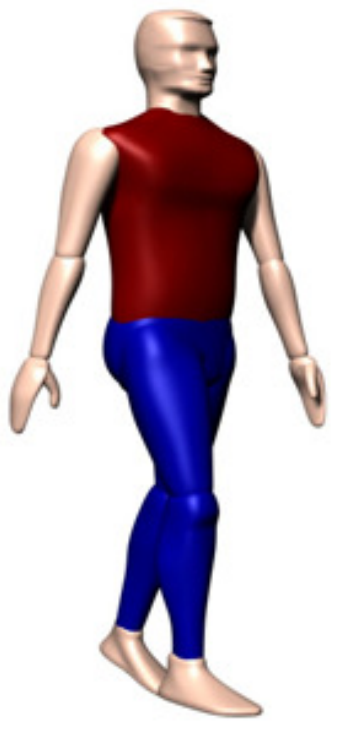

Figure 1. Humanoid model

\subsection{Exoskeleton}

Passive orthosis is widely used to support human movement due to lightweight, compact fitting and easy to operate compared to active orthosis or exoskeletons, but exoskeleton can support human with extra mechanical power to augment their ability to perform daily tasks. The concept of the exoskeleton design in this research is based on supporting people with hemiplegia paralysis to restore gait disorder. The exoskeleton shown in Figure 2 is designed to fit the right side of wearer lower limb and actuate hip, knee and ankle joints. The exoskeleton structure was designed in Solid Works then exported and assembled in Visual Nastran 4D using body tools. Hip, knee and ankle joints of the exoskeleton were set to be parallel in position with humanoid hip, knee and ankle joints and rotate in the sagittal plane ( $\mathrm{Y}$ axis). Revolute motors are used to actuate the exoskeleton hip and knee joints during the simulation process. 

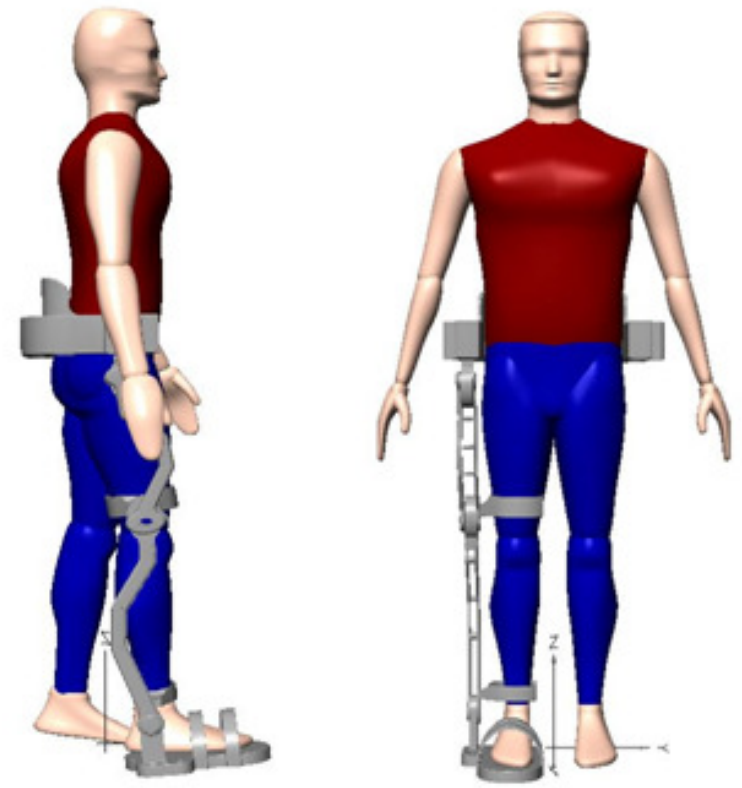

Figure 2. Humanoid and exoskeleton

The mechanical structure of lower limb exoskeleton should follow the characteristics of human lower limb biomechanics such as type of motion, range of motion (RoM), and degree of freedoms (DoF). Table 1 shows the biomechanical properties of human lower limb joints [12], [13]. The exoskeleton joints are controlled according to the biomechanical properties of human lower limb during complete gait cycle to achieve the desired position of each joint.

Table 1. Biomechanical properties of human lower limb joints

\begin{tabular}{|c|c|c|c|}
\hline Joints & DoF & Motion & RoM(deg) \\
\hline Hip & 1 & & $\begin{array}{l}140 \text { (Flexion)/15 } \\
\text { (extension) }\end{array}$ \\
\hline Knee & 1 & & $\begin{array}{l}120-140(\text { Flexion }) / 0- \\
10 \text { (extension) }\end{array}$ \\
\hline Ankle & 1 & & $\begin{array}{l}40-50 \text { (Flexion)/20 } \\
\text { (extension) }\end{array}$ \\
\hline
\end{tabular}




\subsection{Controller}

Human intelligent control system is more advanced than recently developed control systems, therefore it is difficult to design a control system that can match human functions. Furthermore, the level of force generated by human muscles depends on the condition and physical ability of each individual. In case of people with paralysis, muscles may include involuntary strong signals that influence the behaviour of controllers based on biological signals to provide motion support [14]. The control strategy adopted to control the humanoid and exoskeleton is to use predefined trajectory to achieve motion support during walking, based on the trajectory data of human walking provided by clinical gait analysis (CGA) [15].

PD-Fuzzy logic control is considered in this work to control the humanoid and exoskeleton. Fuzzy control is one of the solutions to handle complicated and nonlinear systems when it is difficult to configure an accurate dynamic model, The main advantage of fuzzy systems theory is to approximate system behaviour if the numerical relations of such a system do not exist [16]. Figure 3 shows the fuzzy logic controller. It contains four main blocks: the inputs of the system interpreted in the fuzzification block; the fuzzy-rules block holds information on how to control the system; the appropriate is rule selected and implemented by an interface mechanism block; the fuzzy results are converted into required output signals by the defuzzification block [17].

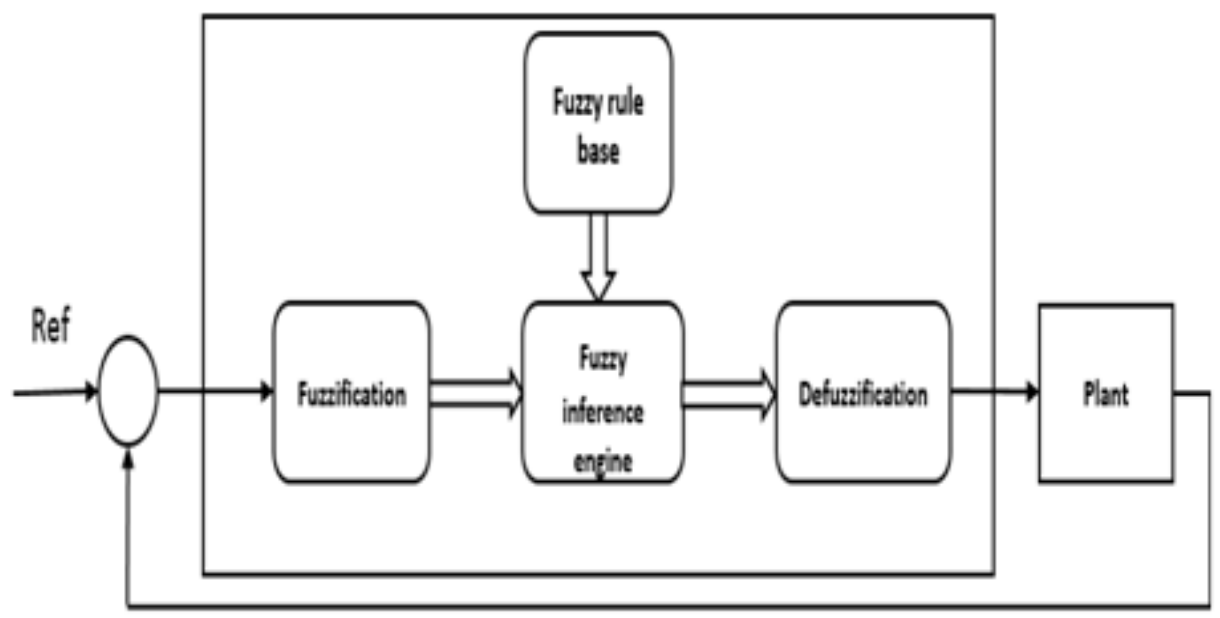

Figure 3. Fuzzy logic control scheme

The fuzzy logic control (FLC) used is based on Mamdani type fuzzy rules with multiple input and single output. The FLC inputs are error $(\mathrm{e})$ and change of error $(\Delta \mathrm{e})$. Five Gaussian membership functions (MF's) used are shown in Table 2: negative small (NS), negative big (NB), zero (Z), positive small (PS) and positive big (PB). The inputs and outputs defined as a result of 25 rules for each fuzzy controller with $50 \%$ overlap between the MF's. Inputs and output are normalised to the range of $[-1,+1]$ as shown in Figure 4. Fuzzy rules are developed to minimise the error and change of error of each input variable. 


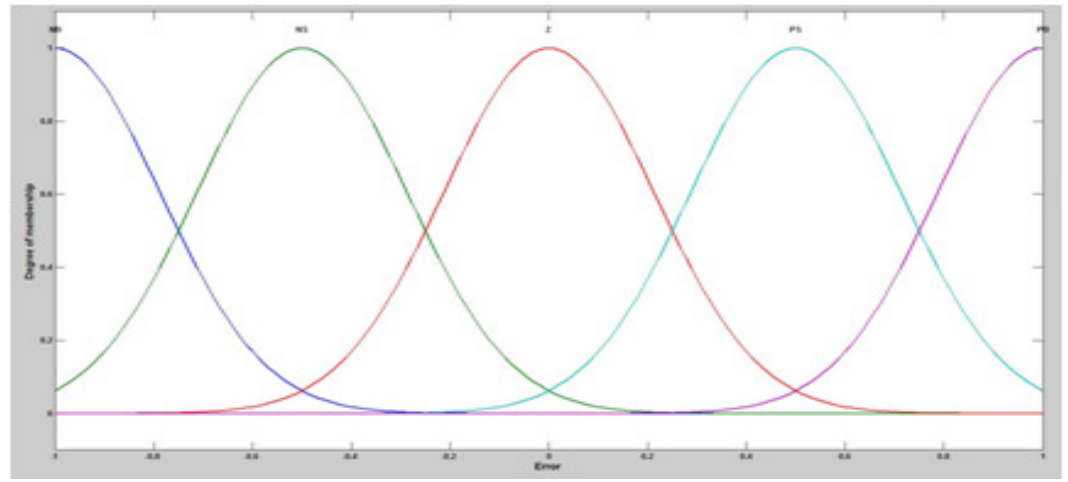

Figure 4. Gaussian membership functions

Table 2. Fuzzy rules

\begin{tabular}{|c|c|c|c|c|c|}
\hline $\mathrm{e} / \Delta \mathrm{e}$ & NS & NB & $\mathbf{Z}$ & PS & PB \\
\hline NS & PB & PB & PS & Z & NS \\
\hline NB & PB & PB & PB & PS & Z \\
\hline Z & PS & PB & Z & NS & NB \\
\hline PS & Z & PS & NS & NB & NB \\
\hline PB & NS & Z & NB & NB & NB \\
\hline
\end{tabular}

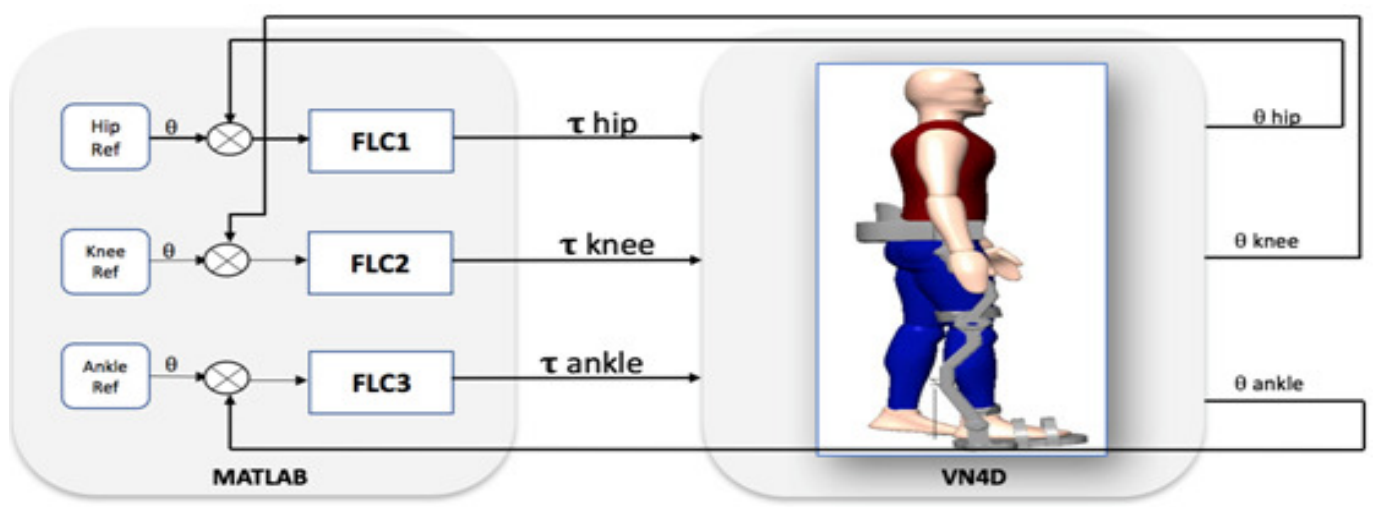

Figure 5. Control system structure

Figure 5 shows the control structure for the exoskeleton and humanoid developed in MATLAB Simulink. The VN4D environment is linked with the controller by adding VN plant from the Simulink library. Three PD-Fuzzy logic controllers shown in Figure 6 (FLC1, FLC2 and FLC3) are used to regulate the input torque $(\boldsymbol{\tau})$ of the right lower limb joints hip, knee and ankle of the humanoid and exoskeleton respectively. The output is formed as orientation (degree) which represents the actual position of each joint, the output is fed back and compared with the reference to determine the error between the actual and the desired position.

The aim of this study is to provide the desired external support by using lower extremity exoskeleton on the affected side to restore natural gait in hemiplegia. Woolley [1] has reported that hemiplegic patients require more metabolic energy cost by $50 \%$ to $67 \%$ compared to normal human while walking at the same speed. A comparison between the torque profiles of hemiplegia and normal human gait cycle of GCA data case was obtained in previous work presented in [3]. The comparison shows that in the hemiplegic case, the torque is on average $25 \%$ to $30 \%$ below 
that of normal human while walking. Consequently, it was assumed that the exoskeleton will provide the hemiplegia patient with $30 \%$ of the torque as compensation to restore the gait and the additional $70 \%$ is the natural torque generated by the patient. Based on this assumption, the output torque of the controller was divided between the humanoid and exoskeleton as shown in Figure 6.

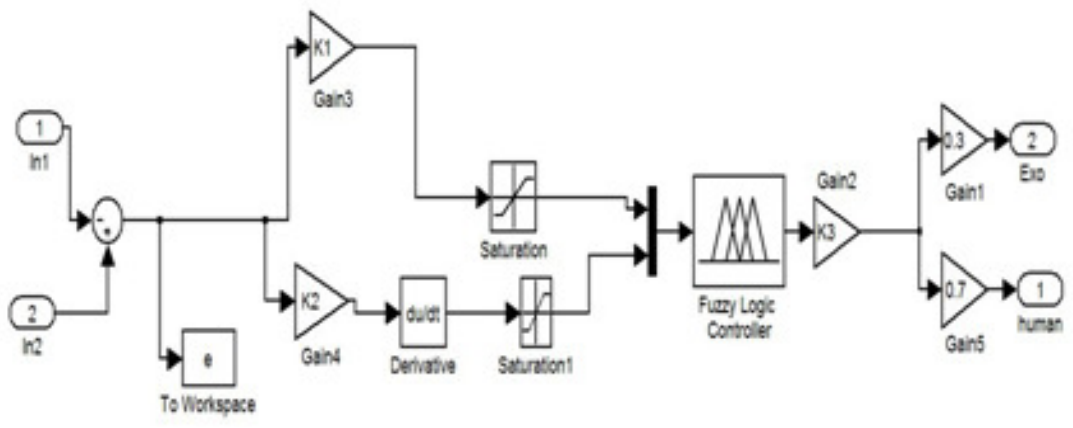

Figure 6. Fuzzy logic controller block (FLC)

\section{SIMULATION RESULTS}

The simulation results shown in Figures 7, 8 and 9 represent the trajectory tracking of humanoid and exoskeleton right joints. Since the left leg of the humanoid is considered as normal, orientation reference is provided to actuate each joint from the left leg to achieve symmetric walking cycle during simulation. The output of each controller represents the orientation of the exoskeleton and humanoid joints during two gait cycles. Figure 7 shows the trajectory tracking of the hip joint position according to the reference and the error during the simulation. It is noted that the PD-Fuzzy controller was able to minimise the error during two gait cycles and achieve smooth tracking. The knee tracking trajectory in Figure 8, shows that the position of humanoid and exoskeleton deviated from the reference at the highest point of the orientation (swing phase of the gait cycle), due to coupling between the humanoid and exoskeleton. Figure 9 shows the trajectory tracking of the ankle joints. It is noted that the ankle joint had more fluctuation and deviation from the reference. The humanoid and exoskeleton feet were not coupled as well as compared to other parts, due to the design mechanism between the exoskeleton foot and the humanoid foot as seen in Figure 2. Therefore, more investigation is needed to enhance the coupling mechanism between the exoskeleton and the humanoid foot.

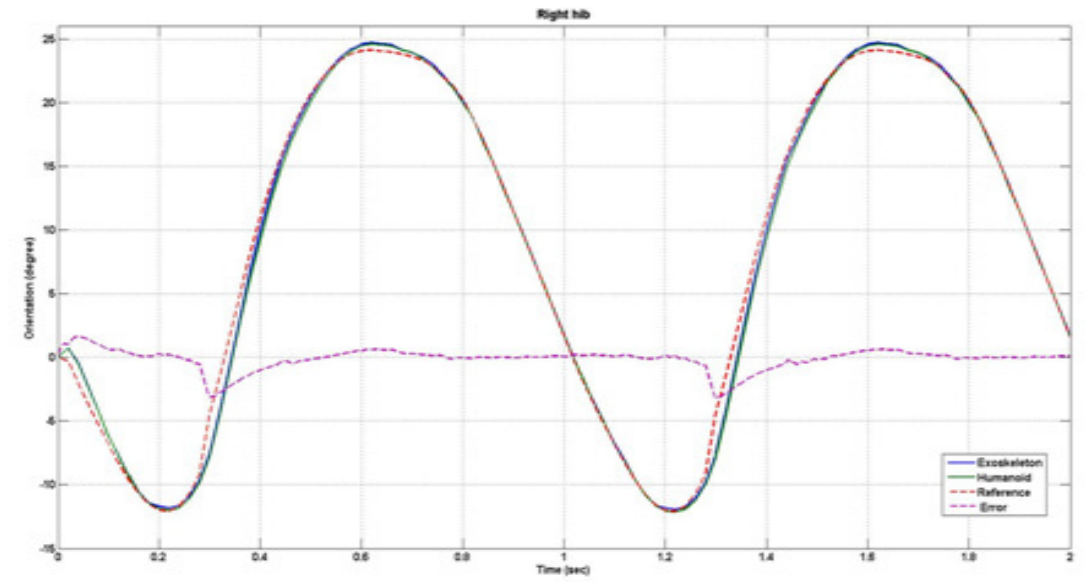

Figure 7. Right hip trajectory tracking 


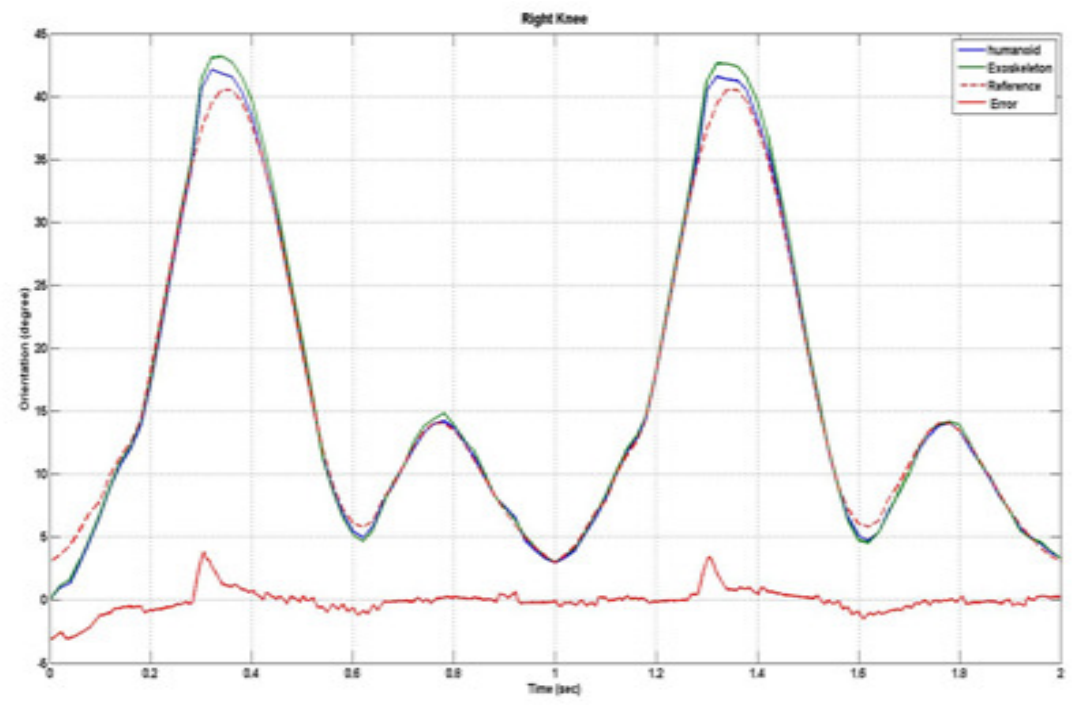

Figure 8. Right knee trajectory tracking

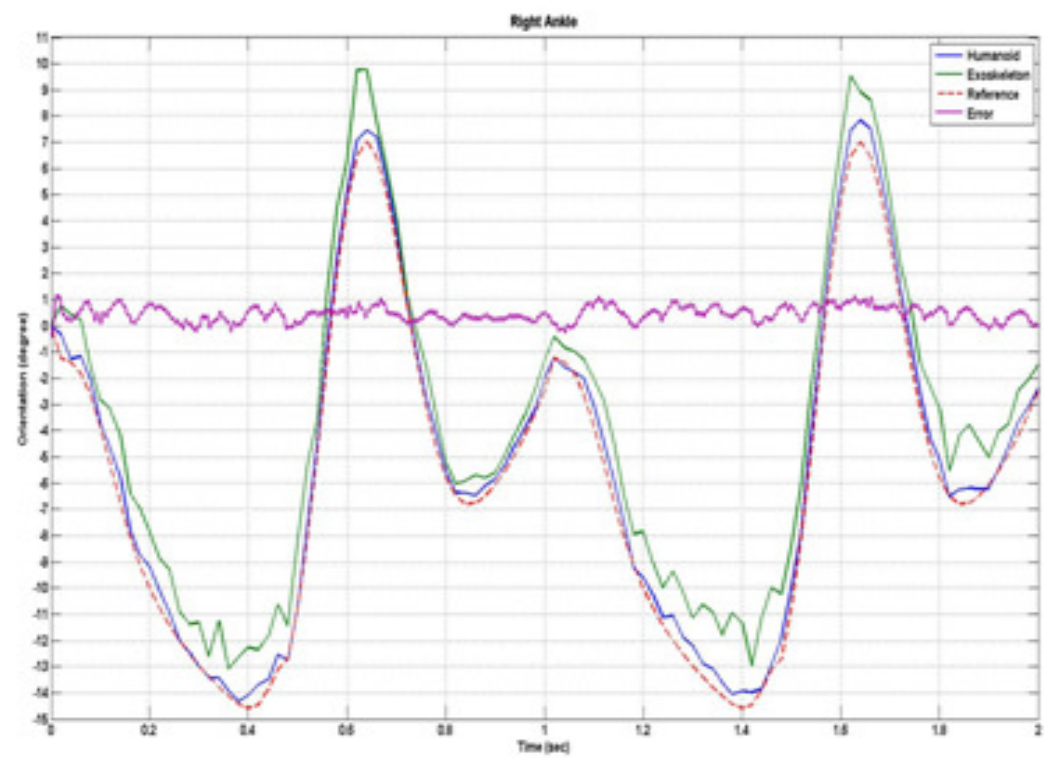

Figure 9. Right ankle trajectory tracking

The investigation carried out constitutes an initial attempt to control the humanoid and exoskeleton to achieve the required support to complete level walking for hemiplegia patient. As noted in the simulation results, the reference signals were not well tracked at some stages of the gait cycle due to the interaction forces generated from the coupled exoskeleton device and humanoid limb. Moreover, the complexity of the system impacted on the response and tracking behaviour during simulation. Therefore, more investigation of the controller approach will be carried out to achieve more robust and stable system performance. The stability of the system will be examined during different locomotion scenarios, such as walking, standing and sitting down. System stability will be examined by applying disturbance forces on humanoid body and the exoskeleton device and the system performance thus achieved will be assessed. 


\section{CONCLUSIONS}

The design and simulation of single leg exoskeleton to support hemiplegia mobility has been presented. The exoskeleton system has been designed to provide $30 \%$ of normal leg torque to augment the available $70 \%$ leg torque and to naturalise the gait of hemiplegic patient. This has been achieved with PD-Fuzzy control mechanism for the hip, knee and ankle joints. Further investigation will look into enhancing the controller and the exoskeleton design in respect of material, fitting and actuation options.

\section{REFERENCES}

[1] S. M. Woolley, "Characteristics of gait in hemiplegia.," Top. Stroke Rehabil., vol. 7, no. 4, pp. 1-18, 2001.

[2] world health organization, "Spinal cord injury," Fact sheet $\mathrm{N}^{\circ} 384,2013$. [Online]. Available: http://www.who.int/mediacentre/factsheets/fs384/en/.

[3] A. Alshatti, M. O. Tokhi, and G. Alrezage, "Design and control of single leg exoskeleton for hemiplegia mobility," in Advances in Cooperative Robotics : Proceedings of the 19th International Conference on CLAWAR 2016, 2016, pp. 832-839.

[4] V. Grosu, C. R. Guerrero, B. Brackx, S. Grosu, B. Vanderborght, and D. Lefeber, "Instrumenting Complex Exoskeletons for Improved Human-Robot Interaction," Instrumentation \& Measurement Magazine, IEEE, no. October, pp. 5-10, 2015.

[5] C.-J. Yang, J.-F. Zhang, Y. Chen, Y.-M. Dong, and Y. Zhang, “A review of exoskeleton-type systems and their key technologies,” Proc. Inst. Mech. Eng. Part C J. Mech. Eng. Sci., vol. 222, no. 8, pp. 1599-1612, 2008.

[6] A. M. Dollar and H. Herr, "Lower extremity exoskeletons and active orthoses: Challenges and stateof-the-art,” IEEE Trans. Robot., vol. 24, no. 1, pp. 144-158, 2008.

[7] A. B. Zoss, H. Kazerooni, and A. Chu, "Biomechanical Design of the Berkeley Lower Extremity Exoskeletong (BLEEX),” IEEE/ASME Trans. Mechatronics, vol. 11, no. 2, pp. 128-138, 2006.

[8] K. a. Strausser and H. Kazerooni, "The development and testing of a human machine interface for a mobile medical exoskeleton,” 2011 IEEE/RSJ Int. Conf. Intell. Robot. Syst., pp. 4911-4916, 2011.

[9] Y. Sankai, "HAL: Hybrid assistive limb based on cybernics," Springer Tracts Adv. Robot., vol. 66, no. STAR, pp. 25-34, 2010.

[10] S. Viteckova, P. Kutilek, and M. Jirina, "Wearable lower limb robotics: A review," Biocybern. Biomed. Eng., vol. 33, no. 2, pp. 96-105, 2013.

[11] D. A. Winter, Biomechanics and motor control of human movement. Hoboken, N.J. : Wiley, c2009, 2009.

[12] K. H. Low, "Robot-assisted gait rehabilitation: From exoskeletons to gait systems," in Defense Science Research Conference and Expo (DSR), 2011, 2011, pp. 1-10.

[13] B. MACKENZIE, "Range of Movement (ROM),” 2004. [Online]. Available: http://www.brianmac.co.uk/musrom.htm. [Accessed: 21-Apr-2016].

[14] H. Kawamoto, T. Hayashi, T. Sakurai, K. Eguchi, and Y. Sankai, "Development of single leg version of HAL for hemiplegia,” Conf Proc IEEE Eng Med Biol Soc, vol. 2009, 2009. 
[15] M. A. Kranzl and D. A. Kopf, “65 year old man with hemiplegia, right side,” 1997. [Online]. Available: http://www.clinicalgaitanalysis.com.

[16] K. M. Passino and S. Yurkovich, Fuzzy Control. California: Addison Wesley Longman, Inc, 1998.

[17] T. J. Ross, Fuzzy Logic with Engineering Applications, 3rd ed. John Wiley \& Sons, 2009. 